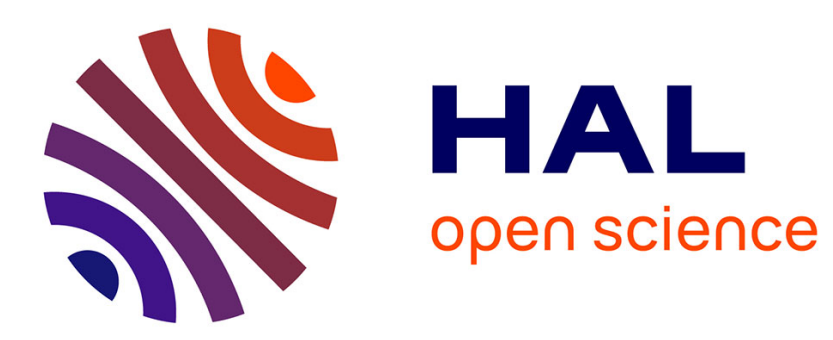

\title{
Adaptation d'un spectromètre de masse à l'analyse des particules instables et à la mesure des pressions d'oxygène élevées
}

B. Weber, B. Bigeard, A. Cassuto

\section{- To cite this version:}

B. Weber, B. Bigeard, A. Cassuto. Adaptation d'un spectromètre de masse à l'analyse des particules instables et à la mesure des pressions d'oxygène élevées. Revue de Physique Appliquée, 1969, 4 (3), pp.373-374. 10.1051/rphysap:0196900403037300 . jpa-00243296

\section{HAL Id: jpa-00243296 https://hal.science/jpa-00243296}

Submitted on 1 Jan 1969

HAL is a multi-disciplinary open access archive for the deposit and dissemination of scientific research documents, whether they are published or not. The documents may come from teaching and research institutions in France or abroad, or from public or private research centers.
L'archive ouverte pluridisciplinaire HAL, est destinée au dépôt et à la diffusion de documents scientifiques de niveau recherche, publiés ou non, émanant des établissements d'enseignement et de recherche français ou étrangers, des laboratoires publics ou privés. 


\title{
ADAPTATION D'UN SPEGTROMÈTRE DE MASSE A L'ANALYSE DES PARTICULES INSTABLES ET A LA MESURE DES PRESSIONS D’OXYGÈNE ÉLEVÉES
}

\author{
Par B. WEBER, B. BIGEARD et A. GASSUTO, \\ Centre de Cinétique Physique et Chimique, route de Vandœuvre, 54-Villers-Nancy. \\ (Reçu le 23 décembre 1968, vévisé le 17 février 1969.)
}

\begin{abstract}
Résumé. - L'ouverture de la chambre d'ionisation, la stabilisation du canon à électrons en émission totale et l'adjonction d'un deuxième collecteur dans un spectromètre de masse Veeco GA 5, nous ont permis de détecter des oxydes volatils, de mesurer des potentiels d'ionisation et des pressions d'oxygène allant jusqu'à $10^{-6}$ torr.
\end{abstract}

Abstract. - With a mass spectrometer Veeco GA 5, by opening the ionization chamber, stabilization of the total emission of the electron gun and adding a second collector, we have been able to analyze volatile oxides, to measure ionization potentials and to measure oxygen pressure down to $10^{-6}$ torr.

Nous avons apporté trois modifications à un spectromètre de masse Veeco type GA 5 dans le but de détecter les particules instables et de mesurer sans inconvénients majeurs, au niveau du multiplicateur, des pressions d'oxygène importantes $\left(10^{-6}\right.$ torr $)$.

1. Ouverture de la chambre d'ionisation. - La première figure indique schématiquement la structure de la chambre d'ionisation avant et après transformation. Le remplacement des parois latérales par de la grille de tungstène, de transparence $95 \%$, permet le passage d'un faisceau moléculaire à travers la source, sans collisions avec les parois et sans perte de sensibilité en fonctionnement normal (pression isotrope). Après transformation, nous avons pu analyser l'oxyde volatil de rhénium $\mathrm{ReO}_{3}$ de même que le rhénium émis par un ruban situé à $10 \mathrm{~cm}$ de la chambre d'ionisation, avec une limite de sensibilité de $10^{11}$ particules $/ \mathrm{cm}^{2} / \mathrm{s}$ émises par le ruban (1/1000 des particules émises traversent la chambre d'ionisation).

\section{Modification du canon à électrons et du système} de stabilisation. - La stabilisation du courant électronique en émission totale, ajoutée à la mise au potentiel

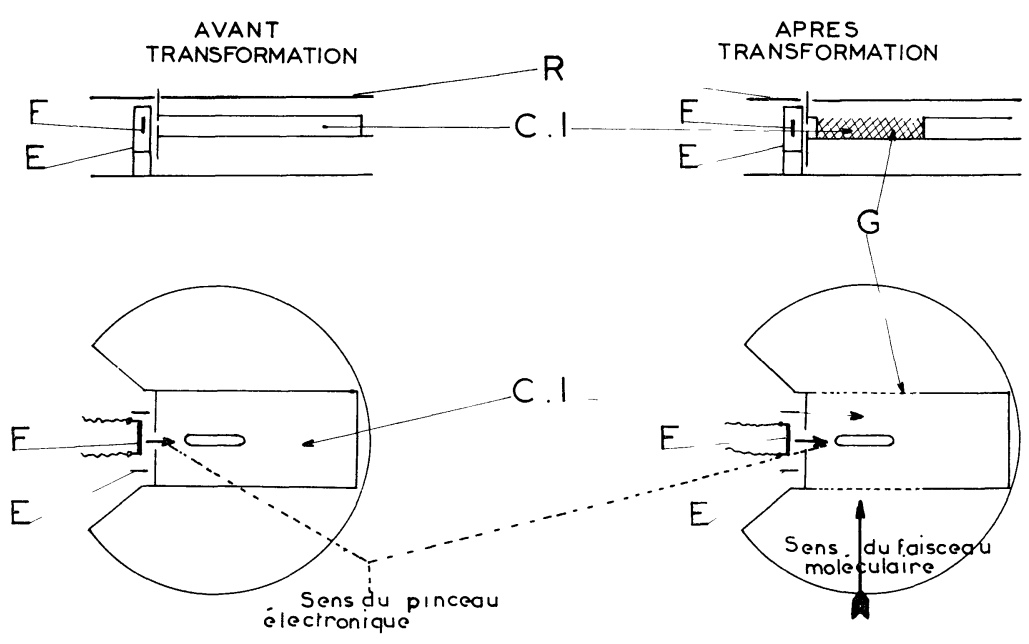

FIG. 1. - Schémas de la source avant et après transformation :

$\mathrm{F}$, filament ; $\mathrm{G}$, grille ; $\mathrm{R}$, repousseur ; E, électrode de pincement ; C.I., chambre d'ionisation. 
de la chambre d'ionisation de l'électrode de pincement prévue par le constructeur (pour une stabilisation par charge d'espace), entraîne une diminution de sensibilité (multipliée par $1 / 5$ pour l'ion $\mathrm{O}_{2}^{+}$), mais permet une mesure approchée des potentiels d'ionisation. La

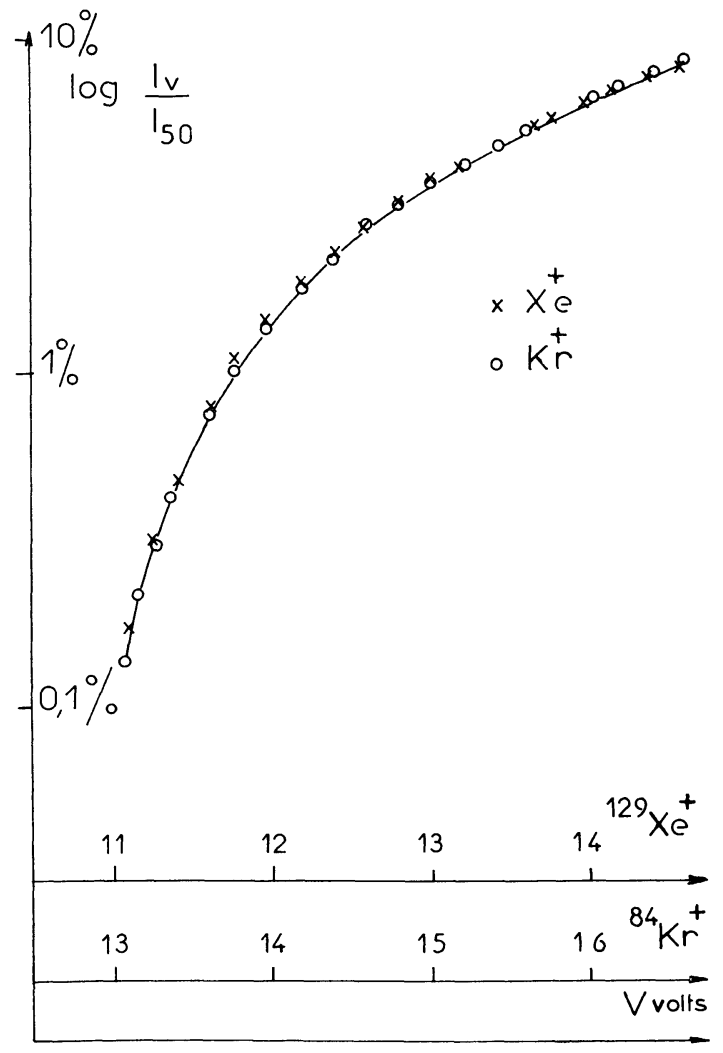

FIG. 2. - Courbes d'ionisation du xénon et du krypton.

figure 2 représente les résultats obtenus par la méthode de Lossing [1] par comparaison des courbes d'ionisation du xénon et du krypton. Les potentiels sont mesurés $\mathrm{au}$ voltmètre numérique. La précision du résultat est de 0,15 volt malgré une décroissance lente des courants et l'absence de champ magnétique de source.

3. Mesure des pressions d'oxygène par adjonction d'un collecteur. - Lorsque les ions $\mathrm{O}_{2}^{+}$tombent sur le multiplicateur, son gain change progressivement.
Un courant d'ions de l'ordre de $10^{-11}$ ampère peut entraîner une multiplication de ce courant par trois en quelques minutes. Mais l'interruption du faisceau s'accompagne d'une désactivation progressive. Afin d'éviter les inconvénients d'un gain variable dans le temps, nous avons placé, parallèlement à la fente d'entrée du multiplicateur, un deuxième collecteur constitué par un filament de molybdène de $3 / 10 \mathrm{de} \mathrm{mm}$, relié à un amplificateur Keithley $610 \mathrm{~B}$. Le décalage entre les deux collecteurs correspond à un décalage d'une unité de masse au voisinage de la masse 32 . La présence du deuxième collecteur ne perturbe pas le fonctionnement du multiplicateur. L'enregistrement simultané des courants reçus par les deux collecteurs donne une mesure relative du gain du multiplicateur pour des ions de nature différente. La mesure des pressions d'oxygène sur le deuxième collecteur n'entraîne aucune activation du multiplicateur. La résolution du spectromètre de masse reste inchangée lorsqu'il est associé au multiplicateur et égale à 85 . Elle est de l'ordre de 50 sur le collecteur additionnel, sans doute en raison d'un léger défaut de parallélisme. La sortie du deuxième collecteur est aisée. Il suffit de le relier au passage additionnel prévu par le constructeur (pour faire varier éventuellement la différence de potentiel entre les deux premières dynodes du multiplicateur lorsque la haute tension reste constante).

La figure 3 indique les résultats obtenus par enregistrement simultané des courants sur les deux collecteurs.

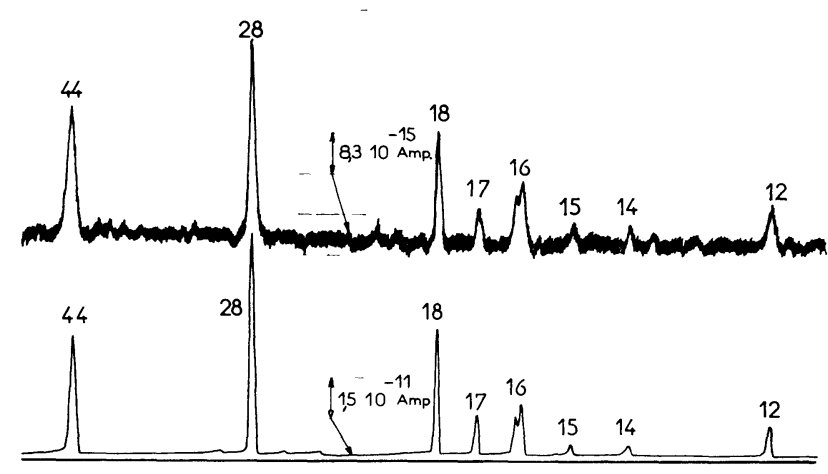

FIG. 3. - Spectres résiduels sur les deux collecteurs (pression résiduelle inférieure à $10^{-9}$ torr, haute tension du multiplicateur $1500 \mathrm{~V}$ ) (échelle supérieure = collecteur additionnel, échelle inférieure $=$ multiplicateur).

\section{BIBLIOGRAPHIE}

[1] Lossing (F. P.), Tickner (A. W.) et Bryce (W. A.), J. Chem. Phys., 1951, 19, 1254. 